\title{
Soliton Taxonomy for a Modification of the Lattice Boussinesq Equation ${ }^{\star}$
}

Jarmo HIETARINTA † and Da-jun ZHANG ${ }^{\S}$

$\dagger$ Department of Physics and Astronomy, University of Turku, FIN-20014 Turku, Finland E-mail: jarmo.hietarinta@utu.fi URL: http://users.utu.fi/hietarin/

‡LPTHE / CNRS / UPMC, 4 place Jussieu 75252 Paris CEDEX 05, France

$\S$ Department of Mathematics, Shanghai University, Shanghai 200444, P.R. China E-mail: djzhang@staff.shu.edu.cn

Received May 24, 2011, in final form July 01, 2011; Published online July 06, 2011 doi:10.3842/SIGMA.2011.061

\begin{abstract}
Integrable multi-component lattice equations of the Boussinesq family have been known for some time. Recently some new equations of this type were found using the Consistency-Around-the-Cube approach. Here we investigate one of these models, B-2, and in particular the consequences of a nonzero deformation parameter $b_{0}>0$, which allows special kinds of solitons in the parameter range $-b_{0} / 3<k<b_{0}$.
\end{abstract}

Key words: lattice Boussinesq equation; integrable lattice equations; solitons; kinks

2010 Mathematics Subject Classification: 35C09, 37K10, 39A14

\section{Introduction}

The lattice Boussinesq equation (lBSQ) reads [1]

$$
\begin{aligned}
& \widetilde{y}-x \widetilde{x}+z=0, \quad \widehat{\widetilde{y}}-\widehat{x} \widehat{\widetilde{x}}+\widehat{z}=0, \\
& \widehat{y}-x \widehat{x}+z=0, \quad \widehat{\widetilde{y}}-\widetilde{x} \widehat{\widetilde{x}}+\widetilde{z}=0, \\
& y-x \widehat{\widetilde{x}}+\widehat{\widetilde{z}}-\frac{p-q}{\widetilde{x}-\widehat{x}}=0,
\end{aligned}
$$

where we have used the standard shorthand notation, e.g., $\widetilde{x}=x_{n+1, m}, \widehat{z}=z_{n, m+1}$, and where $p$ and $q$ are parameters associated with the $n$ and $m$ directions, respectively. Equations (1.1a), (1.1b) are defined on the edges of the elementary square of the Cartesian lattice, while (1.1c) is defined on the square itself. These equations can be naturally extended to a third dimension and the extension is Consistent-Around-the-Cube (CAC) [1]. (For further information about CAC see, e.g., [2, 3, 4].)

The deformation called B-2 in [5] is given by

$$
y-b_{0}(\widehat{\widetilde{x}}-x)-x \widehat{\widetilde{x}}+\widehat{\widetilde{z}}-\frac{p-q}{\widetilde{x}-\widehat{x}}=0
$$

The set (1.1a), (1.1b), (1.1c') also has the CAC property [5]. By changing the sign of $x, p, q$, if necessary, we may assume without loss of generality that $b_{0} \geq 0$. This will be used in the following.

* This paper is a contribution to the Proceedings of the Conference "Symmetries and Integrability of Difference Equations (SIDE-9)" (June 14-18, 2010, Varna, Bulgaria). The full collection is available at http://www.emis.de/journals/SIGMA/SIDE-9.html 
In order to understand the physical meaning of the new parameter $b_{0}$ in $\left(1.1 c^{\prime}\right)$ we construct its soliton solutions. The method is essentially the same as the one given in [6], where we considered the standard IBSQ. We repeat here the salient parts of that construction, concentrating on the differences.

\section{Construction of the one-soliton solution}

In the construction we use the consistency cube of Fig. 1 in an essential way. Here $F=(x, y, z)^{T}$ and $p, q, r$ stand for the direction parameters of the three directions. In practice consistency

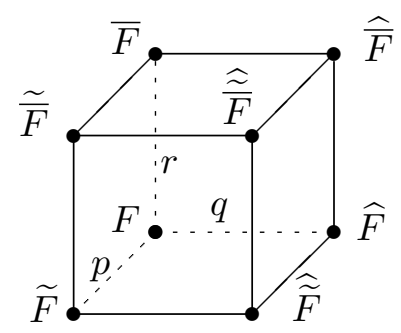

Figure 1. The CAC-cube.

means that the equations on the sides provide a Bäcklund transformation between the bottom and the top equations. In the present case the side equations are

$$
\begin{aligned}
& \bar{y}=x \bar{x}-z, \quad \widetilde{\bar{x}}=\frac{\widetilde{z}-\bar{z}}{\widetilde{x}-\bar{x}}, \quad \widehat{\bar{x}}=\frac{\widehat{z}-\bar{z}}{\widehat{x}-\bar{x}}, \\
& \widetilde{\bar{z}}=b_{0}(\widetilde{\bar{x}}-x)+x \widetilde{\bar{x}}-y+\frac{p-r}{\widetilde{x}-\bar{x}}, \\
& \widehat{\bar{z}}=b_{0}(\widehat{\bar{x}}-x)+x \widehat{\bar{x}}-y+\frac{q-r}{\widehat{x}-\bar{x}},
\end{aligned}
$$

along with some of their shifts.

\subsection{The background solution}

First one has to construct the background solution. This is operationally the same as in [6], but the parameter $b_{0}$ will induce some changes. We follow the fixed-point idea [7] which means that the variables on the bottom and top square are the same. Thus, omitting the bar-shift from (2.1), the equations to solve are

$$
\begin{aligned}
& y=x^{2}-z, \quad \widetilde{z}-z=\widetilde{x}(\widetilde{x}-x), \quad \widehat{z}-z=\widehat{x}(\widehat{x}-x), \\
& \widetilde{z}=b_{0}(\widetilde{x}-x)+x \widetilde{x}-y+\frac{p-r}{\widetilde{x}-x}, \quad \widehat{z}=b_{0}(\widehat{x}-x)+x \widehat{x}-y+\frac{q-r}{\widehat{x}-x} .
\end{aligned}
$$

The solution is given by

$$
\begin{aligned}
& x_{0}=a n+b m+c_{1}, \\
& z_{0}=\frac{1}{2} x_{0}^{2}+\frac{1}{2}\left(a^{2} n+b^{2} m+c_{2}\right)+c_{3}, \\
& y_{0}=\frac{1}{2} x_{0}^{2}-\frac{1}{2}\left(a^{2} n+b^{2} m+c_{2}\right)-c_{3},
\end{aligned}
$$

where we have introduced new parameters $a, b$, which are related to $p, q$ by

$$
a^{3}-b_{0} a^{2}=p-r, \quad b^{3}-b_{0} b^{2}=q-r,
$$


and $c_{1}, c_{2}, c_{3}$ are arbitrary constants (in [6] a different sign convention was used). Here the new parameter $b_{0}$ makes the correspondence slightly more involved.

Since the parameters $a, b$ seem to be the natural ones for this equation we may write $\left(1.1 c^{\prime}\right)$ using them obtaining

$$
y-x \widehat{\widetilde{x}}+\widehat{\widetilde{z}}-\frac{a^{3}-b^{3}}{\widetilde{x}-\widehat{x}}-b_{0}\left[(\widehat{\widetilde{x}}-x)-\frac{a^{2}-b^{2}}{\widetilde{x}-\widehat{x}}\right]=0 .
$$

Now we observe that the $b_{0}$ term is in fact related to lattice potential KdV equation (lpKdV) given by

$$
(\widehat{\widetilde{x}}-x)(\widetilde{x}-\widehat{x})=a^{2}-b^{2}
$$

In other words, $\left(1.1 c^{\prime}\right)$ can be interpreted as a combination of IBSQ (1.1c) and lpKdV.

\subsection{The one-soliton solution}

Next, in order to construct the one-soliton solution (1SS) we again use the CAC-cube, now with variables $x, y, z$ at the bottom square corresponding to the background solution and the bar-shifted variables $\bar{x}, \bar{y}, \bar{z}$ on the top square to the 1SS. Thus, using parametrization (2.3), equations to solve are now

$$
\begin{aligned}
& \bar{y}=x \bar{x}-z, \quad \quad \widetilde{\bar{x}}=\frac{\widetilde{z}-\bar{z}}{\widetilde{x}-\bar{x}}, \quad \widehat{\bar{x}}=\frac{\bar{z}-\widehat{z}}{\bar{x}-\widehat{x}}, \\
& \widetilde{\bar{z}}=b_{0}(\widetilde{\bar{x}}-x)+x \overline{\bar{x}}-y+\frac{a^{3}-k^{3}-b_{0}\left(a^{2}-k^{2}\right)}{\widetilde{x}-\bar{x}}, \\
& \widehat{\bar{z}}=b_{0}(\widehat{\bar{x}}-x)+x \widehat{\bar{x}}-y+\frac{b^{3}-k^{3}-b_{0}\left(b^{2}-k^{2}\right)}{\widehat{x}-\bar{x}},
\end{aligned}
$$

where $k$ is the soliton parameter.

Following the method given in [6] we expand the 1SS as

$$
(\bar{x}, \bar{y}, \bar{z})=\left(\bar{x}_{0}+X, \bar{y}_{0}+Y, \bar{z}_{0}+Z\right)
$$

where $\left(\bar{x}_{0}, \bar{y}_{0}, \bar{z}_{0}\right)$ is the bar-shifted $\left(x_{0}, y_{0}, z_{0}\right)$, i.e.,

$$
\begin{aligned}
& \bar{x}_{0}=a n+b m+k+c_{1}, \\
& \bar{z}_{0}=\frac{1}{2} \bar{x}_{0}^{2}+\frac{1}{2}\left(a^{2} n+b^{2} m+k^{2}+c_{2}\right)+c_{3}, \\
& \bar{y}_{0}=\frac{1}{2} \bar{x}_{0}^{2}-\frac{1}{2}\left(a^{2} n+b^{2} m+k^{2}+c_{2}\right)-c_{3} .
\end{aligned}
$$

With these definitions we find from (2.4a) that $Y=x_{0} X$. Thus we only need to solve for $X, Z$, for which we have from (2.4)

$$
\begin{array}{ll}
\widetilde{X}=\frac{-\overline{\widetilde{x}}_{0} X+Z}{X-(a-k)}, & \widetilde{Z}=\frac{-\left(\widetilde{\bar{z}}_{0}+y_{0}+b_{0} x_{0}\right) X+\left(b_{0}+x_{0}\right) Z}{X-(a-k)}, \\
\widehat{X}=\frac{-\bar{x}_{0} X+Z}{X-(b-k)}, & \widehat{Z}=\frac{-\left(\widehat{\bar{z}}_{0}+y_{0}+b_{0} x_{0}\right) X+\left(b_{0}+x_{0}\right) Z}{X-(b-k)} .
\end{array}
$$

This system can be linearized by taking $(X, Z)=\left(\frac{g}{f}, \frac{h}{f}\right)$, because then we can write it as

$$
\widetilde{\Psi}=N \Psi, \quad \widehat{\Psi}=M \Psi, \quad \Psi=(g, h, f)^{T},
$$


where

$$
N=\left(\begin{array}{ccc}
\tilde{\bar{x}}_{0} & -1 & 0 \\
\widetilde{\bar{z}}_{0}+y_{0}+b_{0} x_{0} & -b_{0}-x_{0} & 0 \\
-1 & 0 & a-k
\end{array}\right), \quad M=\left(\begin{array}{ccc}
\widehat{\bar{x}}_{0} & -1 & 0 \\
\widehat{\bar{z}}_{0}+y_{0}+b_{0} x_{0} & -b_{0}-x_{0} & 0 \\
-1 & 0 & b-k
\end{array}\right)
$$

These matrices satisfy the integrability condition $\widehat{N} M=\widetilde{M} N$. An important observation is that the $N, M$ matrices can both be diagonalized using the matrix $Q$ defined by

$$
Q(n, m)=\left(\begin{array}{ccc}
x_{0}(n, m)+b_{0}-\omega_{2} & -1 & 0 \\
x_{0}(n, m)+b_{0}-\omega_{1} & -1 & 0 \\
\frac{-\left(x_{0}(n, m)+b_{0}-k\right)}{k\left(3 k-2 b_{0}\right)} & \frac{1}{k\left(3 k-2 b_{0}\right)} & 1
\end{array}\right)
$$

because then we have

$$
N=Q(n+1, m)^{-1} D(a) Q(n, m), \quad M=Q(n, m+1)^{-1} D(b) Q(n, m),
$$

where

$$
D(a)=\left(\begin{array}{ccc}
a-\omega_{2} & 0 & 0 \\
0 & a-\omega_{1} & 0 \\
0 & 0 & a-k
\end{array}\right)
$$

The entries $\omega_{i}(\neq k)$ appearing in the above equations are the roots of

$$
W^{3}-k^{3}-b_{0}\left(W^{2}-k^{2}\right)=0 .
$$

This always has the root $\omega_{0}=k$, and if $b_{0}=0$ then $\omega_{i}=k \zeta^{i}, i=1,2$, where $\zeta \neq 1$ is the cubic root of unity. The key observation is that changing $b_{0}$ changes the values of the other roots $\omega_{i}(k)$, and although the $\omega_{i}, i=1,2$ can still be complex conjugates of each other although not of magnitude $k$, we also have the novel possibility that all $\omega_{i}$ can be real. In fact

$$
\omega_{j}(k)=\frac{1}{2}\left[\left(b_{0}-k\right)+(-1)^{j} \sqrt{\left(b_{0}-k\right)\left(b_{0}+3 k\right)}\right], \quad j=1,2 .
$$

So $\omega_{i}$ are real when $-b_{0} / 3<k<b_{0}$. Using the above formulae we can construct $\Psi$ :

$$
\Psi(n, m)=Q(n, m)^{-1} D(a)^{n} D(b)^{m} Q(0,0) \Psi(0,0),
$$

from which we find

$$
\begin{aligned}
& f=\sum_{\nu=0}^{2}\left(a-\omega_{\nu}(k)\right)^{n}\left(b-\omega_{\nu}(k)\right)^{m} \rho_{\nu}^{0}, \\
& g=\sum_{\nu=0}^{2}\left(\omega_{\nu}(k)-k\right)\left(a-\omega_{\nu}(k)\right)^{n}\left(b-\omega_{\nu}(k)\right)^{m} \rho_{\nu}^{0}, \\
& h=x_{0} g+\sum_{\nu=0}^{2}\left(\omega_{\nu}(k)^{2}-k^{2}\right)\left(a-\omega_{\nu}(k)\right)^{n}\left(b-\omega_{\nu}(k)\right)^{m} \rho_{\nu}^{0} .
\end{aligned}
$$

Here, instead of $g_{00}, h_{00}, f_{00}$, we have introduced new constants $\rho_{\nu}^{0}$, which are defined by

$$
\rho_{0}^{0}=f_{00}-\frac{g_{00}\left(c_{1}+b_{0}-k\right)-h_{00}}{\left(\omega_{1}-k\right)\left(\omega_{2}-k\right)}, \quad \rho_{\nu}^{0}=\frac{g_{00}\left(c_{1}+b_{0}-\omega_{\nu}\right)-h_{00}}{(-1)^{\nu}\left(\omega_{1}-\omega_{2}\right)\left(\omega_{\nu}-k\right)}, \quad \nu=1,2 .
$$


Using (2.5), (2.6) we can recover the 1SS as

$$
\begin{aligned}
& x^{1 \mathrm{SS}}=x_{0}+\frac{\omega_{0} \rho_{0}+\omega_{1} \rho_{1}+\omega_{2} \rho_{2}}{\rho_{0}+\rho_{1}+\rho_{2}}, \\
& z^{1 \mathrm{SS}}=z_{0}+x_{0} \frac{\omega_{0} \rho_{0}+\omega_{1} \rho_{1}+\omega_{2} \rho_{2}}{\rho_{0}+\rho_{1}+\rho_{2}}+\frac{\omega_{0}^{2} \rho_{0}+\omega_{1}^{2} \rho_{1}+\omega_{2}^{2} \rho_{2}}{\rho_{0}+\rho_{1}+\rho_{2}}, \\
& y^{1 \mathrm{SS}}=y_{0}+x_{0} \frac{\omega_{0} \rho_{0}+\omega_{1} \rho_{1}+\omega_{2} \rho_{2}}{\rho_{0}+\rho_{1}+\rho_{2}} .
\end{aligned}
$$

Here $x_{0}, y_{0}, z_{0}$ were defined in $(2.2)$ and

$$
\rho_{\nu}(n, m)=\left(a-\omega_{\nu}(k)\right)^{n}\left(b-\omega_{\nu}(k)\right)^{m} \rho_{\nu}^{0}, \quad \nu=0,1,2 .
$$

The $\rho_{\nu}$ correspond to the plane-wave factors (PWF) in the continuous case and we use the same name here.

\section{The different types of solitons}

\subsection{Generic properties}

The soliton (2.10), when expressed in variables $x, y, z$, has a linearly or quadratically growing background part (2.2). In order to show the soliton behavior we ignore this part and only discuss the part

$$
U:=k+\frac{g}{f}=\frac{\omega_{0} \rho_{0}+\omega_{1} \rho_{1}+\omega_{2} \rho_{2}}{\rho_{0}+\rho_{1}+\rho_{2}} .
$$

From (3.1) we can immediately see that the soliton is a kink or anti-kink and that in the asymptotic region it will level off to some $\omega_{i}$, depending on the term that dominates in the particular asymptotic region of $n, m$. In comparing the terms $i$ and $j$ it is useful to determine the line where they are equal, it is given by

$$
n \log \left|\frac{a-\omega_{i}}{a-\omega_{j}}\right|+m \log \left|\frac{b-\omega_{i}}{b-\omega_{j}}\right|=0 .
$$

Then the $i$ term will dominate in the growing $n$ region with respect to this line, if $\left|a-\omega_{i}\right|>$ $\left|a-\omega_{j}\right|$, etc. Furthermore one has to verify that the $i=j$ term is dominating over the $k$ term, and therefore only a half-line is relevant. In the generic case one needs to draw three such half-lines, which results in three different asymptotic regions. Examples of these will be given below.

\subsection{The behavior of solitons for $b_{0}=0$}

Let us first consider the classical case for which $b_{0}=0$. Then $\omega_{\nu}=k \zeta^{\nu}$ where $\zeta=-\frac{1}{2}(1+i \sqrt{3})$. For simplicity let us assume that $0<k<a, b$. We have

$$
\left|a-\omega_{\nu}\right|=\sqrt{a^{2}+a k+k^{2}} \quad \text { for } \quad \nu=1,2,
$$

from which it follows that $\left|\rho_{1}\right|=\left|\rho_{2}\right|$. Furthermore let us define

$$
\varphi_{a}(k)=\arccos \left(\frac{a+k / 2}{\sqrt{a^{2}+a k+k^{2}}}\right)
$$

so that $a-\omega_{\nu}=\sqrt{a^{2}+a k+k^{2}} e^{i \varphi_{a}(k)}$. Since $\omega_{1}^{*}=\omega_{2}$ we can have real solitons if we choose $\left(\rho_{2}^{0}\right)^{*}=\rho_{1}^{0}=\alpha e^{i \beta}$; without loss of generality we can also take $\rho_{0}^{0}=1$. 

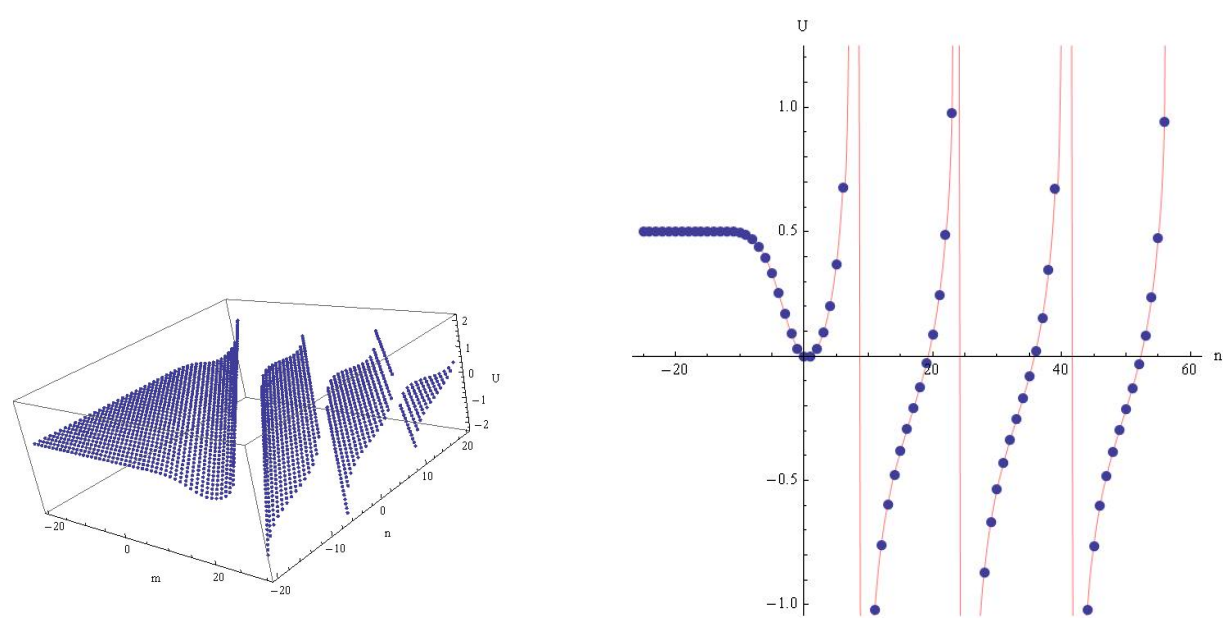

Figure 2. Typical oscillation for the $b_{0}=0$ case. On the left a $3 \mathrm{D}$ view of $U$ of (3.1), on the right a section at $m=0$. The parameters have values $b_{0}=0, a=b=2, k=0.5, \rho_{\nu}^{0}=1$.

We can now write the soliton part of $(3.1)$ in the $b_{0}=0$ case as

$$
U=k \frac{A^{n} B^{m}+2 \alpha \cos \left(\xi-\frac{2}{3} \pi\right)}{A^{n} B^{m}+2 \alpha \cos \xi},
$$

where

$$
A=\frac{a-k}{\sqrt{a^{2}+a k+k^{2}}}, \quad B=\frac{b-k}{\sqrt{b^{2}+b k+k^{2}}}, \quad \xi=n \varphi_{a}(k)+m \varphi_{b}(k)+\beta .
$$

Since $0<k<a, b$ we have $0<A, B<1$. Thus $A^{n} B^{m} \rightarrow+\infty$ as $n, m \rightarrow-\infty$ and therefore in that direction $U \rightarrow k$. On the other hand, $A^{n} B^{m} \rightarrow 0$ as $n, m \rightarrow+\infty$ and the behavior in that direction is oscillatory since the cosine terms dominate. In the continuous case there would be some values of the independent variables for which the denominator actually vanishes. In the discrete case we can only say that for large enough $n, m$ we can choose their particular values so that $\xi$ gets arbitrarily close to $(N+1 / 2) \pi$. And then due to the extra $-2 \pi / 3$ in the numerator, $U$ can take arbitrarily large (positive or negative) values. Thus in the $b_{0}=0$ case all single-soliton solutions are effectively singular asymptotically. This is illustrated in Fig. 2.

\subsection{The behavior of solitons when $k<-b_{0} / 3$ or $b_{0}<k\left(b_{0}>0\right)$}

The solitons behave essentially the same way as for $b_{0}=0$ also in the $b_{0} \neq 0$ case, provided that $k>b_{0}$ or $k<-b_{0} / 3$, i.e., $\left(k-b_{0}\right)\left(b_{0}+3 k\right)>0$. The roots $\omega_{i}, i=1,2$ are still complex conjugates, with $\left|\omega_{i}\right|^{2}=k\left(k-b_{0}\right)$, and $\left|\rho_{1}\right|=\left|\rho_{2}\right|$. The detailed expressions for variables $A, B$, $\varphi_{a}(k)$, used in (3.2), will just have a different form. Let us assume $b_{0}<k<a, b$, then we have

$$
\begin{aligned}
& \omega_{0}=k, \quad \omega_{1}=\frac{1}{2}\left[b_{0}-k-i \sqrt{\left(k-b_{0}\right)\left(b_{0}+3 k\right)}\right], \\
& \omega_{2}=\frac{1}{2}\left[b_{0}-k+i \sqrt{\left(k-b_{0}\right)\left(b_{0}+3 k\right)}\right], \\
& \left|a-\omega_{i}\right|=\sqrt{a^{2}+a k+k^{2}-b_{0}(a+k)} \quad \text { for } i=1,2, \\
& \varphi_{a}(k)=\arccos \left(\frac{a+k / 2-b_{0} / 2}{\sqrt{a^{2}+a k+k^{2}-b_{0}(a+k)}}\right) .
\end{aligned}
$$

For the plane-wave factors we have rations

$$
A=\frac{a-k}{\left|a-\omega_{1}\right|}, \quad B=\frac{b-k}{\left|b-\omega_{1}\right|}, \quad A^{2}=1+\frac{a\left(b_{0}-k\right)+k\left(b_{0}-2 a\right)}{a^{2}+a k+k^{2}-b_{0}(a+k)}<1,
$$



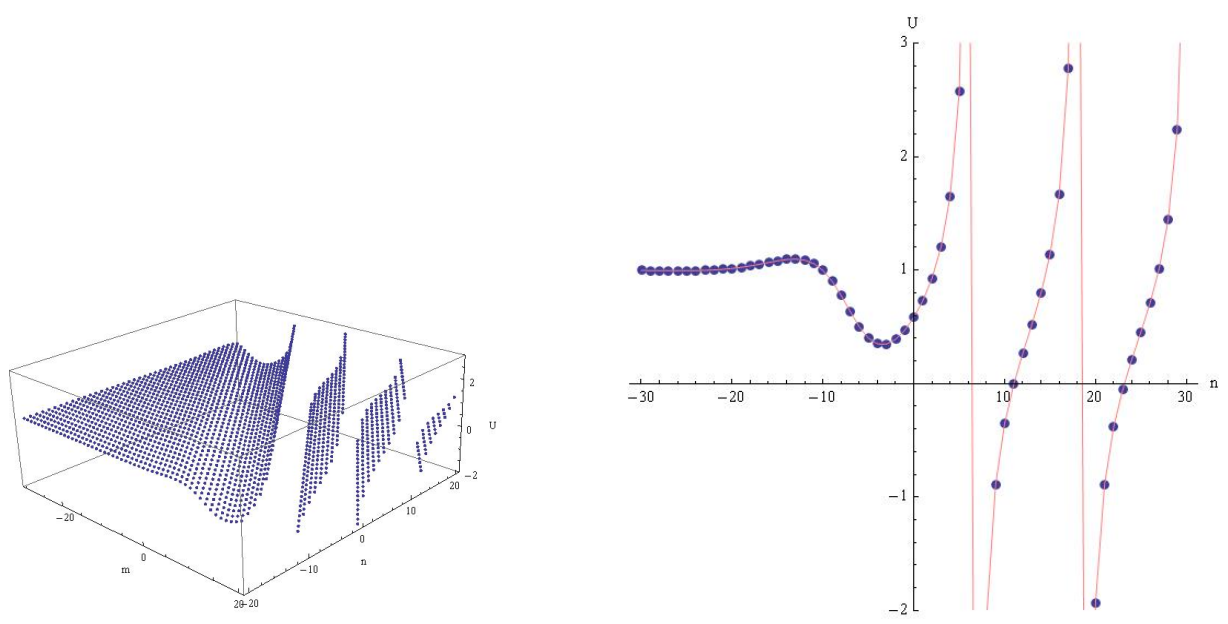

Figure 3. Oscillatory behavior of $U$ for $b_{0}=1, a=b=4, \rho_{\nu}^{0}=1, k=1.75, m=0$.

or more generally

$$
\left|\frac{a-\omega_{\nu}(k)}{a-\omega_{0}(k)}\right|^{2}=\frac{a^{3}-k^{3}-b_{0}\left(a^{2}-k^{2}\right)}{(a-k)^{3}} \quad(>0) .
$$

One example of such a soliton is given in Fig. 3.

\subsection{The behavior of solitons when $-b_{0} / 3<k<b_{0}$}

If $-b_{0} / 3<k<b_{0}$ the situation changes completely, because the roots $\omega_{\nu}(k)$ are now all real, and different in magnitude

$$
\begin{aligned}
& \omega_{0}=k, \quad \omega_{1}=\frac{1}{2}\left[b_{0}-k-\sqrt{\left(b_{0}-k\right)\left(b_{0}+3 k\right)}\right], \\
& \omega_{2}=\frac{1}{2}\left[b_{0}-k+\sqrt{\left(b_{0}-k\right)\left(b_{0}+3 k\right)}\right] .
\end{aligned}
$$

Now different terms of $U$ can dominate depending on the value of $k$.

For the elements appearing in the PWF's We find

$$
\begin{array}{lll}
-\frac{1}{3} b_{0}<k<0: & k<w_{1}<w_{2}, & a-k>a-w_{1}>a-w_{2}, \\
0<k<\frac{2}{3} b_{0}: & w_{1}<k<w_{2}, & a-w_{1}>a-k>a-w_{2}, \\
\frac{2}{3} b_{0}<k<b_{0}: & w_{1}<w_{2}<k, & a-w_{1}>a-w_{2}>a-k .
\end{array}
$$

The relationships of the middle column above can be seen if we plot $k, w_{1}, w_{2}$ together, see Fig. 4.

There are now a variety of soliton types depending on the values of soliton parameters $k, \omega_{i}$ with respect to the equation parameters $a, b$. All the PWF's are positive, if $a, b>\max \left(k, w_{2}\right)$, and if $a, b<\min \left(k, w_{1}\right)$ the negative sign can be canceled from all terms. In these cases there will be no oscillations. If however $a$ and/or $b$ falls between $\min \left(k, w_{1}\right)$ and $\max \left(k, w_{2}\right)$ then there will be oscillations and furthermore there can be a greater variety in dominant asymptotic behavior. We will next discuss some of the possible cases.

\subsection{1 $a, b>\max \left(k, w_{2}\right)$ or $a, b<\min \left(k, w_{1}\right)$}

If $a, b>\max \left(k, w_{2}\right)$ all $\mathrm{PWF}$ are positive. The term that dominates for large positive values of $n, m$ is $(a-k)^{n}(b-k)^{m}$ for (3.3a) and $\left(a-w_{1}\right)^{n}\left(b-w_{1}\right)^{m}$ in all other cases. For large negative 


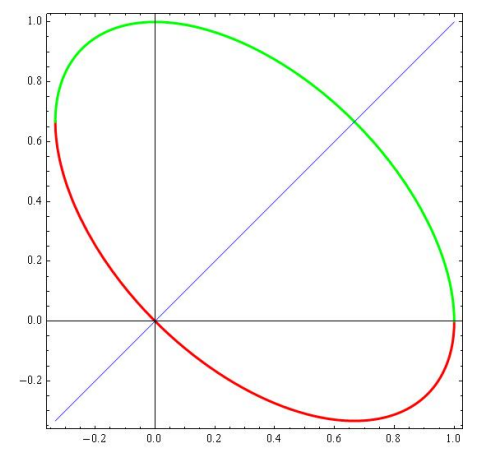

Figure 4. The roots of (2.7) as functions of $k$ for $b_{0}=1: w_{2}$ in green, $w_{1}$ in red, and $\omega_{0}=k$ in blue.
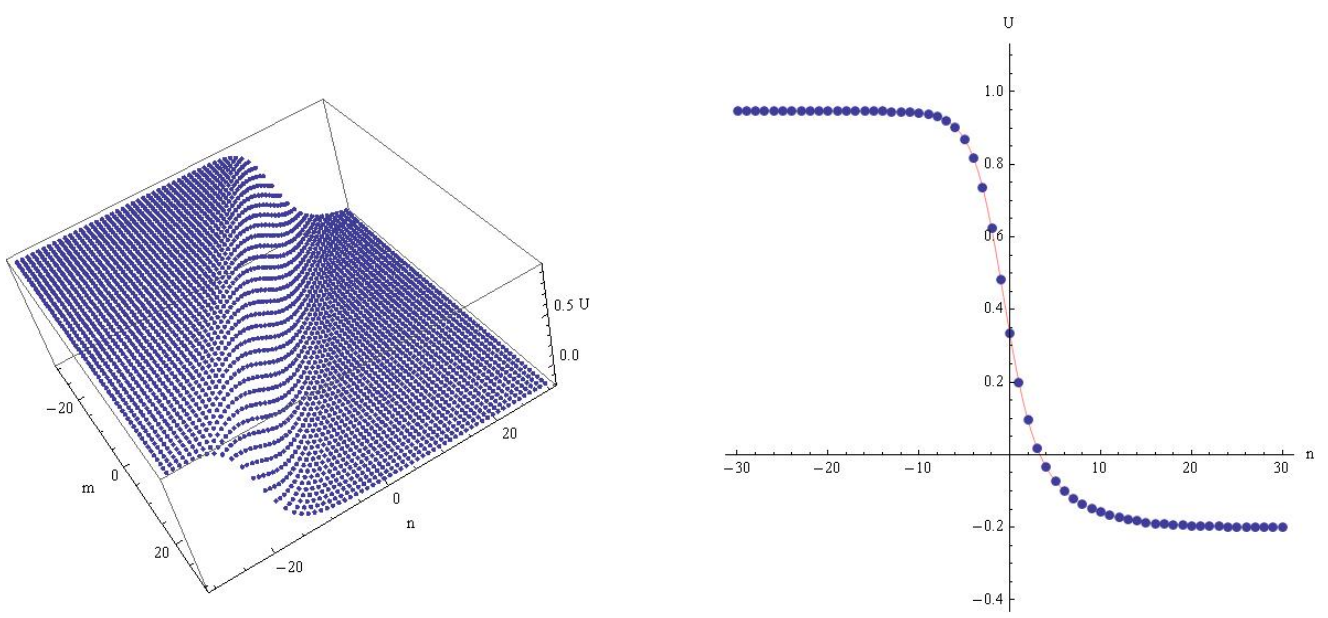

Figure 5. When $a, b \geq \max \left(k, \omega_{2}\right)$ the soliton looks like a simple anti-kink. $b_{0}=1, k=-0.2, a=2$, $b=3, \rho_{\nu}^{0}=1$.

values for both $n, m$ the dominating term is $(a-k)^{n}(b-k)^{m}$ for $(3.3 \mathrm{c})$ and $\left(a-w_{2}\right)^{n}\left(b-w_{2}\right)^{m}$ in all other cases. For other $n, m$ directions we must compare values more closely. Furthermore, choosing a relatively large $\rho_{i}^{0}$ will make the corresponding term dominate in some finite region, although not asymptotically.

In the example of Fig. 5 the $a, b$ values are so large in comparison with $k$ that the solution looks like a simple anti-kink. Similarly for large negative values of $a, b$ we get a simple kink.

If $a, b$ are only moderately larger than $b_{0}$ the double soliton nature of the kink starts to appear. This is illustrated in Fig. 6. A kink is shown in Fig. 7.

\subsection{2 $\omega_{1}<a, b<\omega_{0}, \omega_{2}$}

For this choice the PWF's $\rho_{0}$ and $\rho_{2}$ have alternating signs as functions of $n, m$, but $\rho_{1}>0$. This means that at the line where $\left|\rho_{0}\right|=\rho_{1}$ or $\left|\rho_{2}\right|=\rho_{1}$ there will be unbounded oscillations in the asymptotic regions, while the behavior is smooth around the line $\left|\rho_{0}\right|=\left|\rho_{2}\right|$. One such case is given in Fig. 8, for which the parameters are $b_{0}=1, k=0.5, a=0.2, b=0.3, \rho_{\nu}^{0}=1$. It then follows that $\omega_{0}=0.5, \omega_{1}=-0.309, \omega_{2}=0.809$ and $a-\omega_{0}=-0.3, b-\omega_{0}=-0.2$, $a-\omega_{1}=0.509, b-\omega_{1}=0.609, a-\omega_{2}=-0.609, b-\omega_{2}=-0.509$. The oscillatory transition lines are at $n=-0.475 m$ for $\left|\rho_{0}\right|=\left|\rho_{1}\right|$ and $n=m$ for $\left|\rho_{2}\right|=\left|\rho_{1}\right|$, while the transition line $\left|\rho_{0}\right|=\left|\rho_{2}\right|$ at $n=-0.758 \mathrm{~m}$ is smooth. Another example is illustrated in Fig. 9, the opening angle to the lowest level is now smaller. 

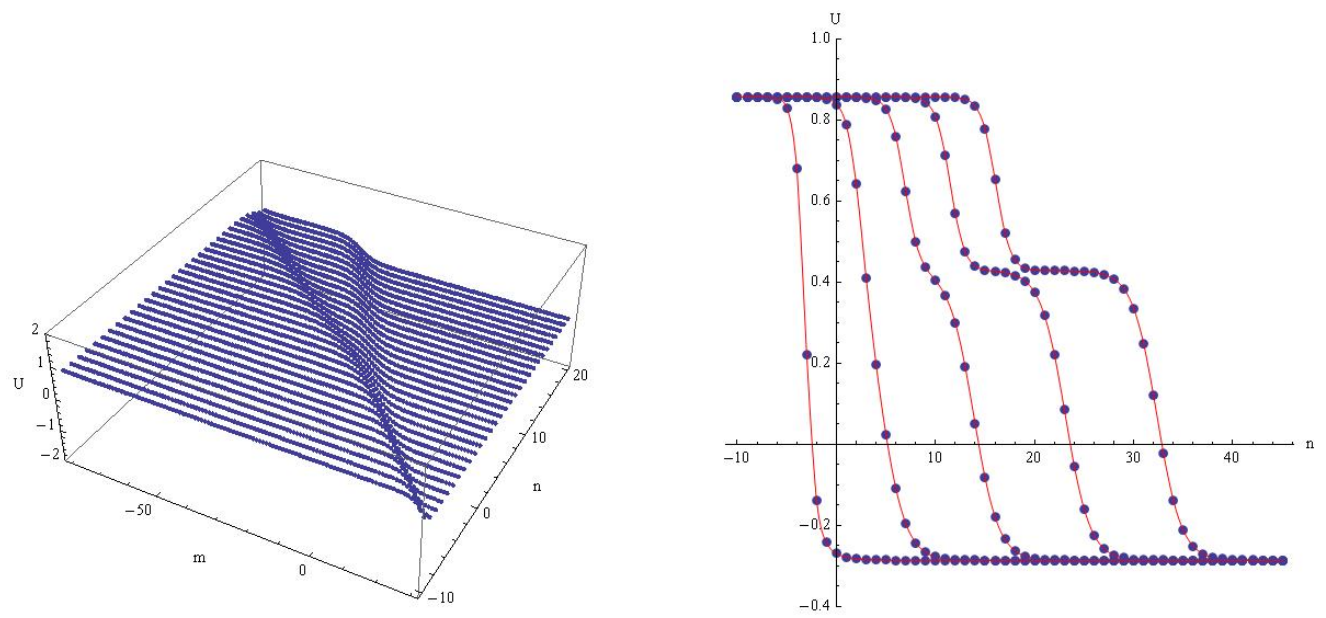

Figure 6. A $3 \mathrm{D}$ view and sections for $m=-70,-50,-30,-10,10$ of an antikink with $b_{0}=1, k=\frac{3}{7}$, $a=1, b=2, \rho_{\nu}^{0}=1$. All $a-\omega_{i}, b-\omega_{i}$ are positive.
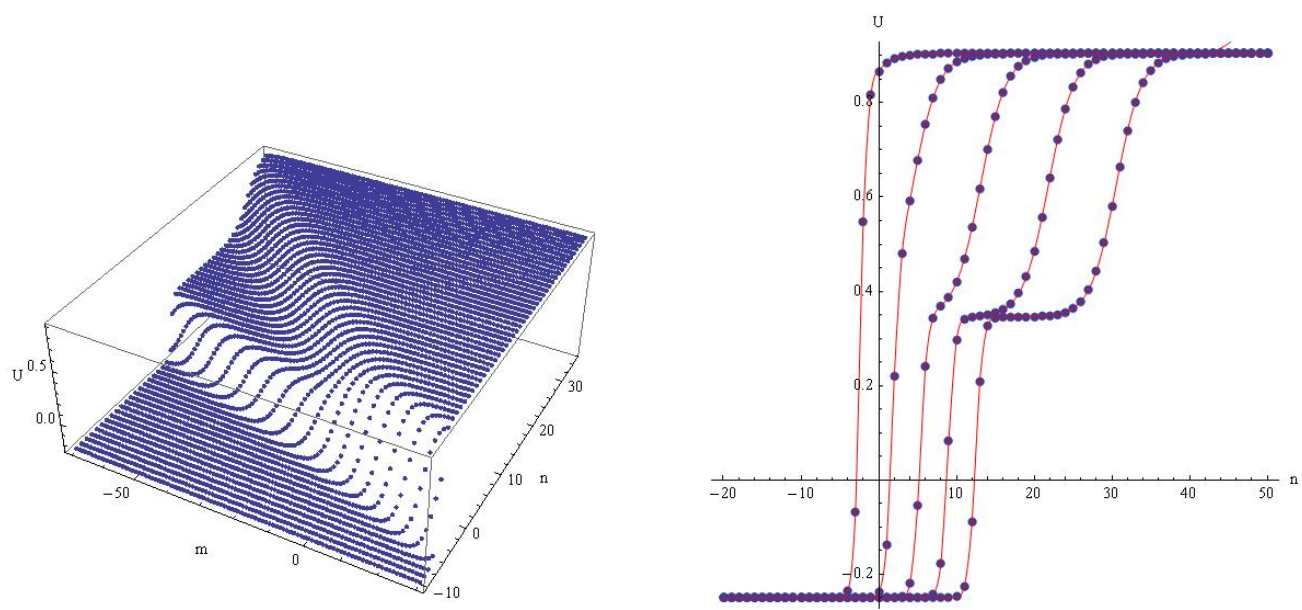

Figure 7. A $3 \mathrm{D}$ view and sections for $m=-70,-50,-30,-10,10$ of a kink with $b_{0}=1, k=-0.25$, $a=-0.3, b=-1.5, \rho_{\nu}^{0}=1$. All $a-\omega_{i}, b-\omega_{i}$ are negative.

\subsection{3 $\omega_{1}<a<\omega_{0}<b<\omega_{2}$}

The behavior is still more diverse when $a<\omega_{0}<b$. This is illustrated in Fig. 10, where the parameters are $b_{0}=1, k=0.5, a=-0.18, b=0.63$. The parameters $b_{0}, k, \omega_{i}$ are the same as in Section 3.4.2 but since now $b>\omega_{0}$ the signs are different: $a-\omega_{0}=-0.68, b-\omega_{0}=0.131$, $a-\omega_{1}=0.129, b-\omega_{1}=0.939, a-\omega_{2}=-0.989, b-\omega_{2}=-0.179$. Now all the transition lines are oscillatory, the line $\left|\rho_{0}\right|=\left|\rho_{1}\right|$ oscillates as a function of $n$, the lines $\left|\rho_{0}\right|=\left|\rho_{2}\right|$ and $\left|\rho_{1}\right|=\left|\rho_{1}\right|$ as functions of $m$.

\subsection{4 $\omega_{0}=\omega_{2}$}

Finally we have an example for $\omega_{0}=\omega_{2}$. This leads to a pure kink or antikink solution, because $\rho_{0}=\rho_{2}$. This happens, e.g., for $b_{0}=1, k=2 / 3$. Since $a-\omega_{0}<0$ but $a-\omega_{1}>0$ we have oscillations on the transition region. This is illustrated in Fig. 11. 

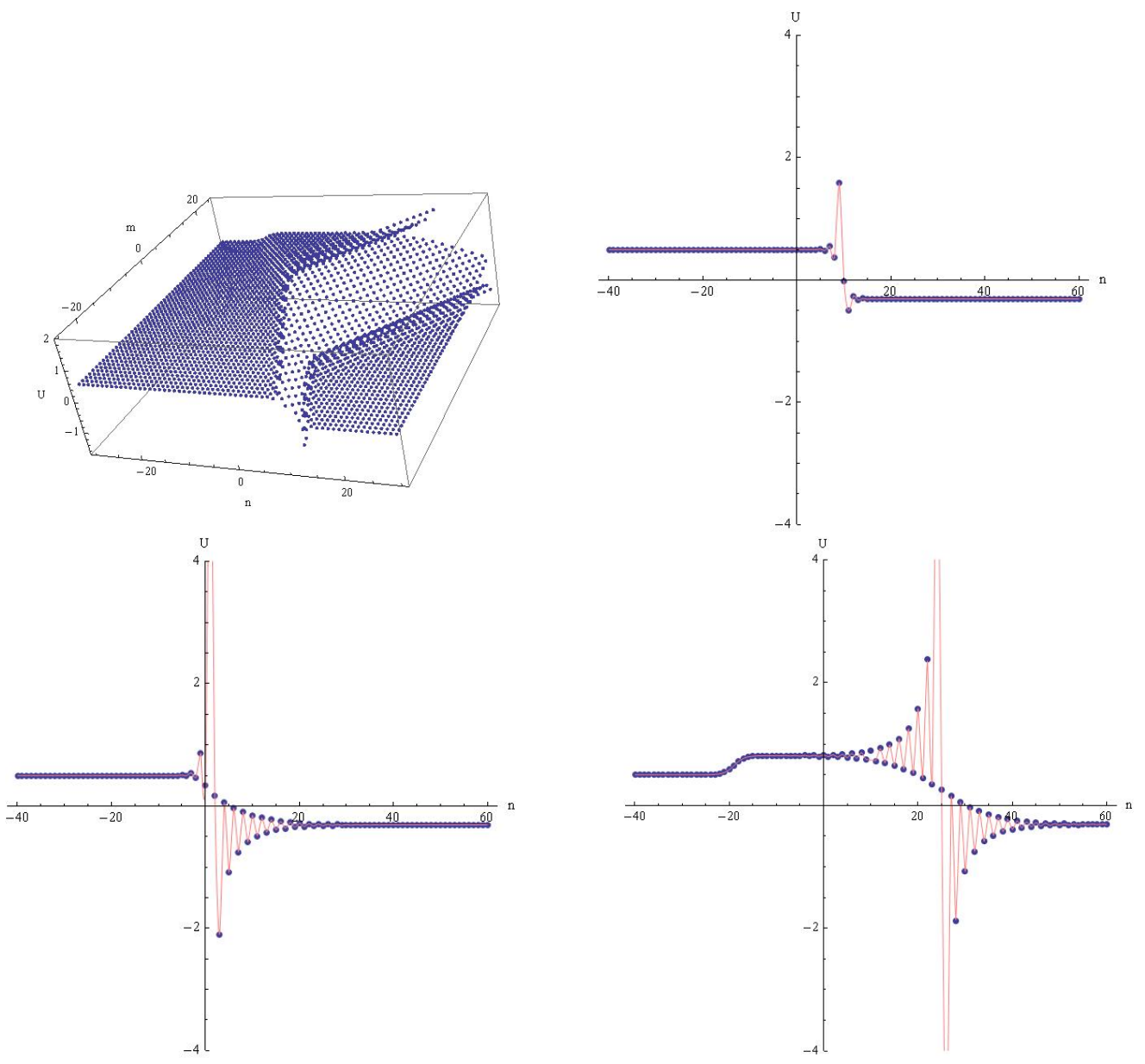

Figure 8. Three-level 1SS with oscillations on the boundary of level $\omega_{1}$. $3 \mathrm{D}$ view with cross sections at $m=-20,0,25$. The parameters are $b_{0}=1, k=0.5, a=0.2, b=0.3, \rho_{\nu}^{0}=1$.
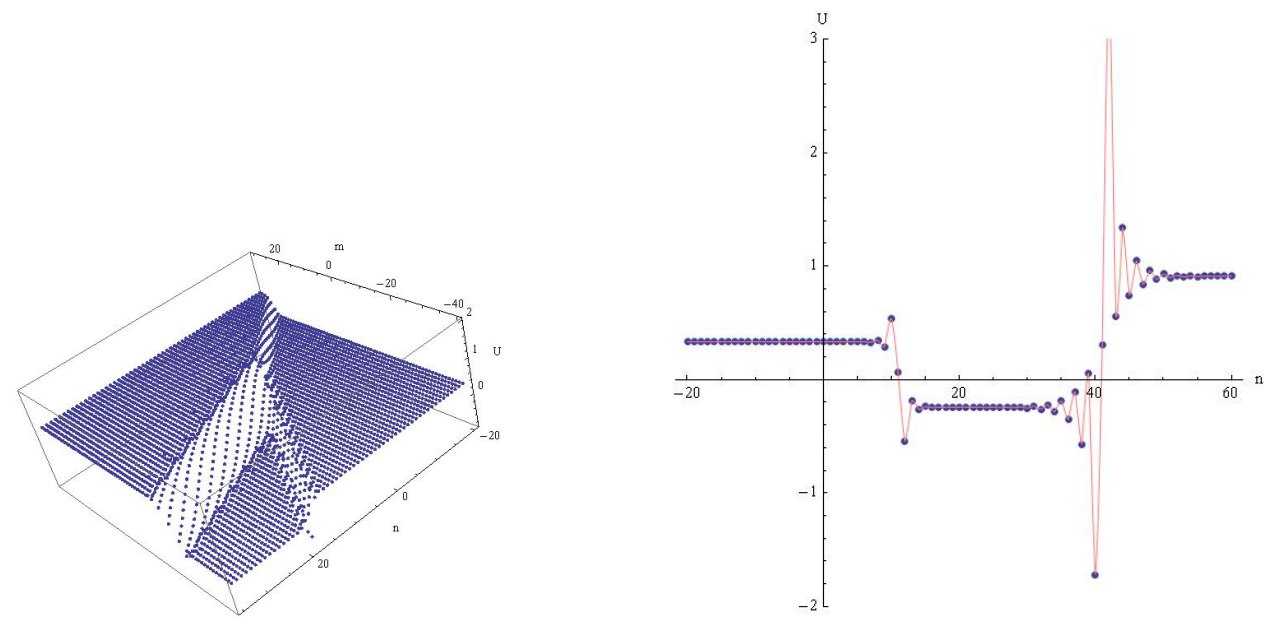

Figure 9. 3D view with cross section at $m=-25$. The parameters are $b_{0}=1, k=\frac{1}{3}, a=0.2, b=0.12$, $\rho_{\nu}^{0}=1$. 

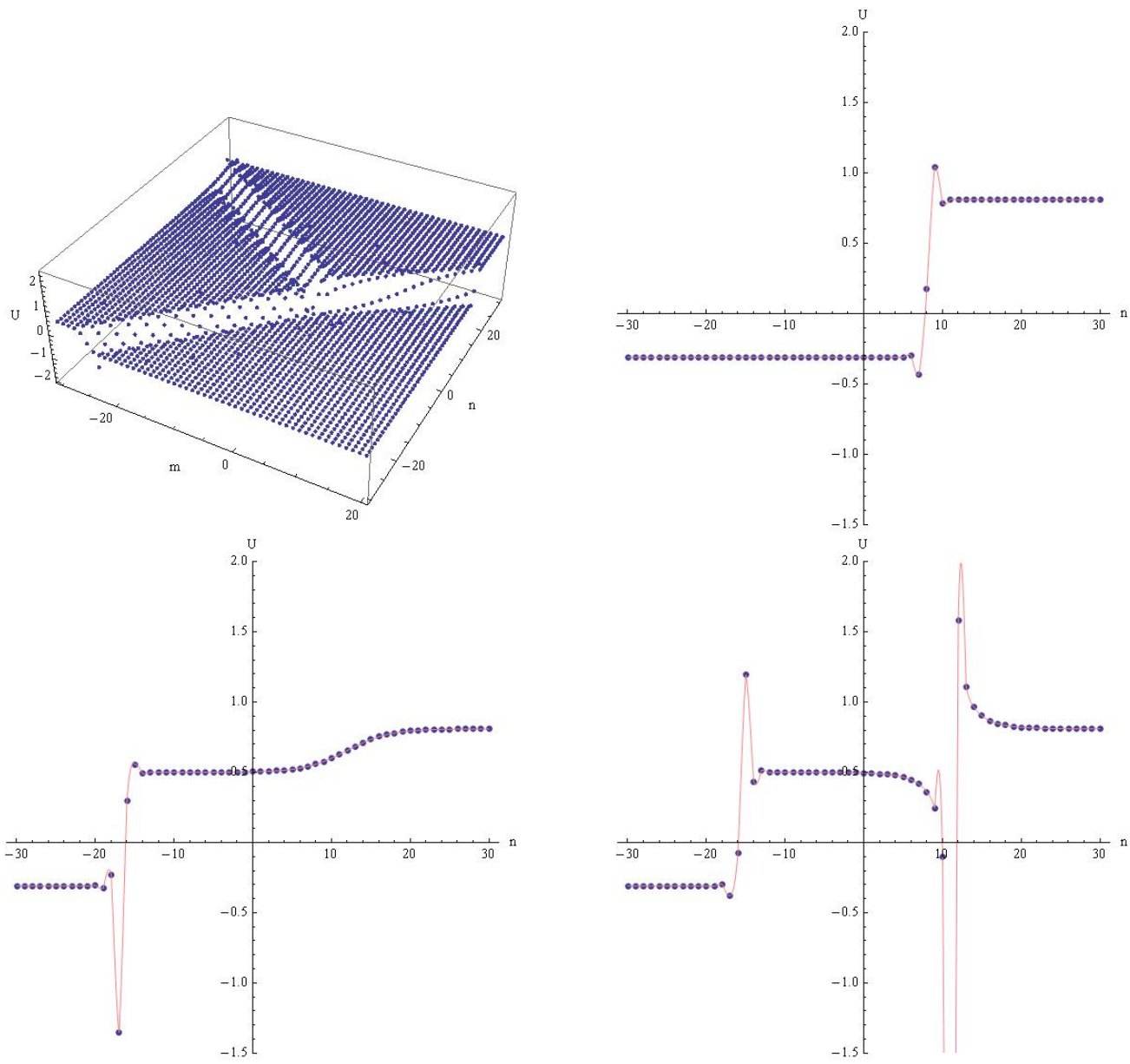

Figure 10. Soliton solution with parameter values $b_{0}=1, k=0.5, a=-0.18, b=0.63, \rho_{\nu}^{0}=1$. Above: $3 \mathrm{D}$ view with a section at $m=10$, below: two adjacent sections at $m=-14,-13$. The levels are, from left, 1, 0 and 2. The 0-2 transition line is smooth in $n$ for even $m$ and oscillatory in $n$ for odd $m$.

\section{Bilinearization, Casoratians and $N$-soliton solutions}

Above we have discussed only the 1SS. Although the figures look complicated one can obtain the multi-soliton solutions using a procedure similar to [6]. We only list the main results

By the dependent variable transformation

$$
x=x_{0}-\frac{g}{f}, \quad z=z_{0}-x_{0} \frac{g}{f}+\frac{h}{f}, \quad y=y_{0}-x_{0} \frac{g}{f}+\frac{s}{f},
$$

we can bilinearize the B-2 lattice consisting of $H B 1=(1.1 \mathrm{a}), H B 2=(1.1 \mathrm{~b}), H B 3=\left(1.1 c^{\prime}\right)$ as

$$
\begin{aligned}
H B 1 & =\frac{\mathcal{B}_{1}}{f \widetilde{f}}, \quad H B 2=\frac{\mathcal{B}_{2}}{f \widehat{f}}, \\
H B 3 & =\frac{\mathcal{B}_{3} \mathcal{B}_{4}+(a-b) f \widetilde{\widetilde{f}} \mathcal{B}_{4}+\left[a^{2}+a b+b^{2}-b_{0}(a+b)\right] \widetilde{f} \widehat{f} \mathcal{B}_{3}}{(\widetilde{x}-\widehat{x}) f \widetilde{f} \widehat{f} \widehat{\widetilde{f}}},
\end{aligned}
$$

where we have also used the parametrization (2.3), and where the bilinear equations are

$$
\begin{aligned}
\mathcal{B}_{1} & =\widetilde{f}(h+a g)-\widetilde{g}(g+a f)+f \widetilde{s}=0, \\
\mathcal{B}_{2} & =\widehat{f}(h+b g)-\widehat{g}(g+b f)+f \widehat{s}=0,
\end{aligned}
$$



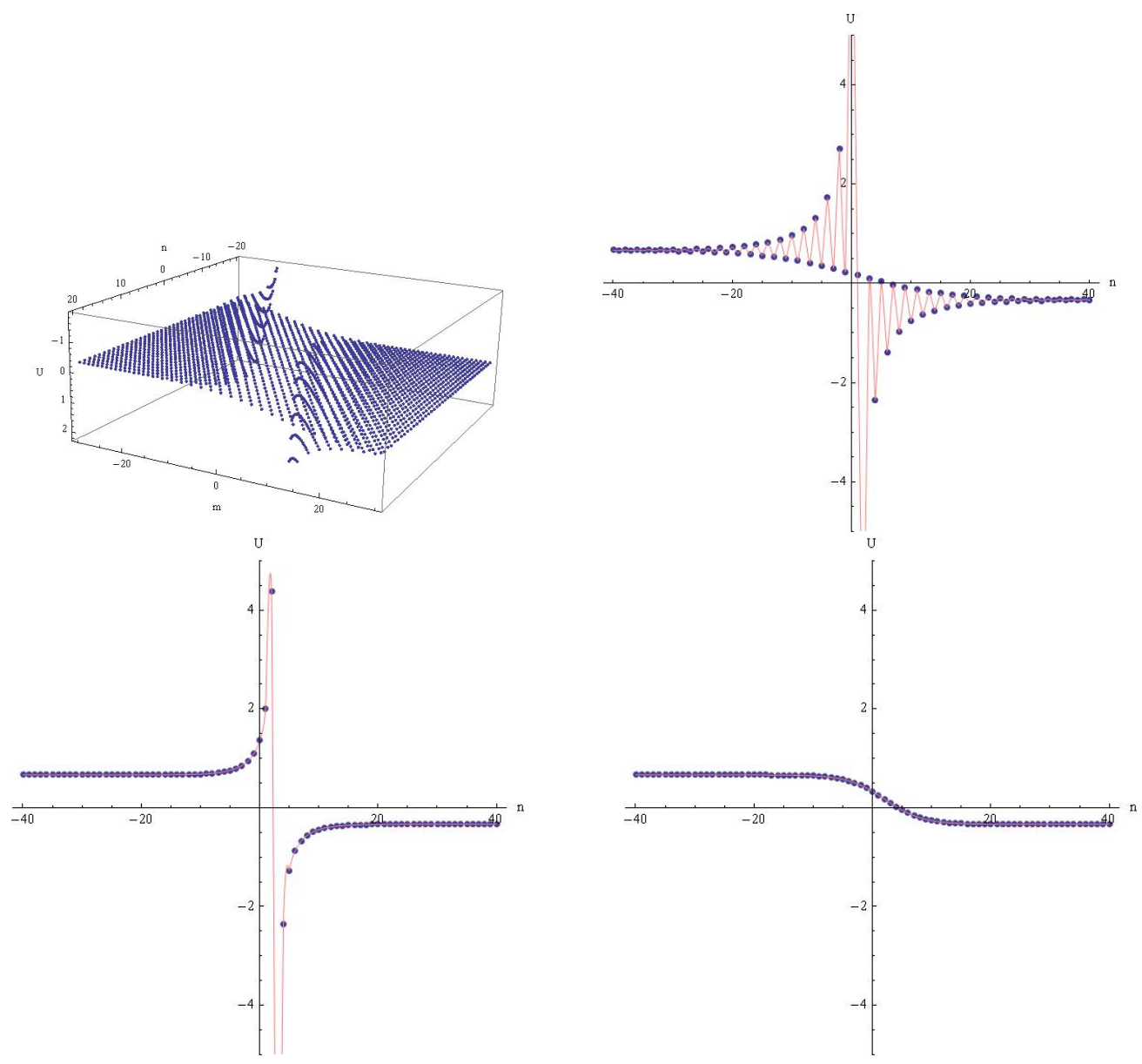

Figure 11. A kink with oscillations. Here $b_{0}=1, k=2 / 3, a=0.2, b=0.12$ and therefore $\rho_{0}=\rho_{2}=$ $(-1)^{n+m}\left|\rho_{2}\right|$, while $\rho_{1}>0$. Top: $3 \mathrm{D}$ view and a section at $m=-3$, bottom: a section with $n+m=1$ on the left and $n+m=0$ on the right.

$$
\begin{aligned}
\mathcal{B}_{3}= & \tilde{f} \widehat{g}-\widehat{f} \widetilde{g}+(a-b)(\widetilde{f} \widehat{f}-f \widehat{\widetilde{f}})=0, \\
\mathcal{B}_{4}= & {\left[a^{2}+a b+b^{2}-b_{0}(a+b)\right](f \widehat{\widetilde{f}}-\widetilde{f} \widehat{f}) } \\
& +\left(a+b-b_{0}\right)(\widehat{\widetilde{f}} g-f \widehat{\widetilde{g}})+\widehat{\widetilde{f}} s+f \widetilde{\widetilde{h}}-g \widehat{\widetilde{g}}=0 .
\end{aligned}
$$

The set of bilinear equations (4.2) admits $N$-soliton solutions in the following Casoratian form,

$$
f=|\widehat{N-1}|, \quad g=|\widehat{N-2}, N|, \quad h=|\widehat{N-2}, N+1|, \quad s=|\widehat{N-3}, N-1, N|,
$$

composed of $\psi=\left(\psi_{1}, \psi_{2}, \ldots, \psi_{N}\right)^{T}$ with

$$
\psi_{j}(n, m, l)=\sum_{s=0}^{2} \varrho_{j, s}^{(0)}\left(-\omega_{s}\left(k_{j}\right)\right)^{l}\left(a-\omega_{s}\left(k_{j}\right)\right)^{n}\left(b-\omega_{s}\left(k_{j}\right)\right)^{m},
$$

where $\omega_{0}(k) \equiv k, \omega_{i}(k)$ for $i=1,2$ are defined in $(2.8)$ and $\varrho_{j, s}^{(0)}$ are constants. Here a $N$ th order Casoratian of the column vector

$$
\psi(n, m, l)=\left(\psi_{1}(n, m, l), \psi_{2}(n, m, l), \ldots, \psi_{N}(n, m, l)\right)^{T}
$$

w.r.t. shift variable $l$, is defined by

$$
C_{n, m}\left(\psi ;\left\{l_{i}\right\}\right)=\left|\psi\left(n, m, l_{1}\right), \psi\left(n, m, l_{2}\right), \ldots, \psi\left(n, m, l_{N}\right)\right| .
$$


Usually we write this in the shorthand notation $[8]\left|l_{1}, l_{2}, \ldots, l_{N}\right|$, and in particular for consecutive sequences we use $\widehat{M} \equiv 0,1, \ldots, M$.

The proof for $(4.2 \mathrm{~d})$ is similar to the one in [6]. In fact, the Casoratian column vector $\psi$ defined in (4.4) satisfies the shift relation

$$
\begin{aligned}
& \widetilde{\psi}-\bar{\psi}=a \psi, \quad \widehat{\psi}-\bar{\psi}=b \psi, \\
& \Gamma \psi=\overline{\bar{\psi}}+b_{0} \overline{\bar{\psi}},
\end{aligned}
$$

where $\Gamma$ is some $N \times N$ matrix. We note that the relation (4.5a) is the same as the one in [6], and it is sufficient for proving the first three equations in (4.2), so here we do not consider them further. The relation (4.5b) is different from the one in [6]. Using it one can generate an explicit form for the identity $(\operatorname{Tr}(\Gamma) f) f=(\operatorname{Tr}(\Gamma) f) f$ (c.f., Appendix of $[6])$,

$$
\begin{aligned}
a^{N-2} \underset{\sim}{f}[ & |\widehat{N-5}, N-3, N-2, N-1, \psi(N-2)|-\mid \widehat{N-4}, N-2, N, \underset{\sim}{(N-2) \mid} \\
& \left.+|\widehat{N-3}, N+1, \psi(N-2)|+b^{N+1} \underset{\sim}{f}+g-b f+b_{0} b^{N-2}(\underset{\sim}{s}-\underset{\sim}{h})\right] \\
- & b^{N-2} \underset{\sim}{f}[|\widehat{N-5}, N-3, N-2, N-1, \underset{\sim}{\psi}(N-2)|-|\widehat{N-4}, N-2, N, \underset{\sim}{\psi}(N-2)| \\
& \left.+|\widehat{N-3}, N+1, \underset{\sim}{\psi}(N-2)|+a^{N+1} \underset{\sim}{f}+g-a f+b_{0} a^{N-2}(\underset{\sim}{s}-\underset{\sim}{h})\right]=0,
\end{aligned}
$$

by which one can then verify $(4.2 \mathrm{~d})$.

\section{Conclusions}

In this paper we have discussed the various soliton solutions of the recently discovered [5] deformation $\left(1.1 c^{\prime}\right)$ of the lattice Boussinesq equation (1.1). It is well known that for Boussinesq-type equations the soliton solution is formed by a combination of three plane waves, but in the standard case with $b_{0}=0$ the parts merge and produce a kink with oscillations. For $b_{0} \neq 0$ the three components are more independent and the solutions look like resonating two-kink solutions with three different asymptotic levels. Depending on the choice of parameters there can be oscillations on the transition region between levels.

\section{Acknowledgments}

One of the authors $(\mathrm{JH})$ was partially supported by Ville de Paris within the "Research in Paris" program. DJZ was supported by the NSFC (No. 11071157). This project is also partially supported by the Shanghai Leading Academic Discipline Project (No. J50101).

\section{References}

[1] Tongas A., Nijhoff F., The Boussinesq integrable system: compatible lattice and continuum structures, Glasg. Math. J. 47 (2005), no. A, 205-219, nlin.SI/0402053.

[2] Bobenko A.I., Suris Y.B., Integrable systems on quad-graphs, Int. Math. Res. Not. 2002 (2002), no. 11, 573-611, nlin.SI/0110004.

[3] Nijhoff F.W., Walker A.J., The discrete and continuous Painlevé VI hierarchy and the Garnier systems, Glasg. Math. J. 43 (2001), no. A, 109-123, nlin.SI/0001054.

[4] Nijhoff F.W., Lax pair for the Adler (lattice Krichever-Novikov) system, Phys. Lett. A 297 (2002), 49-58, nlin.SI/0110027.

[5] Hietarinta J., Boussinesq-like multi-component lattice equations and multi-dimensional consistency, J. Phys. A: Math. Theor. 44 (2011), 165204, 22 pages, arXiv:1011.1978. 
[6] Hietarinta J., Zhang D.J., Multisoliton solutions to the lattice Boussinesq equation, J. Math. Phys. 51 (2010), 033505, 12 pages, arXiv:0906.3955.

[7] Atkinson J., Hietarinta J., Nijhoff F., Seed and soliton solutions of Adler's lattice equation, J. Phys. A: Math. Theor. 40 (2007), F1-F8, nlin.SI/0609044.

[8] Freeman N.C., Nimmo J.J.C., Soliton solutions of the Korteweg-de Vries and Kadomtsev-Petviashvili equations: the Wronskian technique, Phys. Lett. A 95 (1983), 1-3. 